\title{
Respiratory microbes present in the nasopharynx of children hospitalised with suspected pulmonary tuberculosis in Cape Town, South Africa
}

Felix S. Dube ${ }^{1 *}$, Mamadou Kaba ${ }^{1,2}$, F. J. Lourens Robberts ${ }^{1}$, Lemese Ah Tow ${ }^{1}$, Sugnet Lubbe ${ }^{3}$, Heather J. Zar ${ }^{4,5}$ and Mark P. Nicol ${ }^{1,2,6}$

\begin{abstract}
Background: Lower respiratory tract infection in children is increasingly thought to be polymicrobial in origin. Children with symptoms suggestive of pulmonary tuberculosis (PTB) may have tuberculosis, other respiratory tract infections or co-infection with Mycobacterium tuberculosis and other pathogens. We aimed to identify the presence of potential respiratory pathogens in nasopharyngeal (NP) samples from children with suspected PTB.

Method: NP samples collected from consecutive children presenting with suspected PTB at Red Cross Children's Hospital (Cape Town, South Africa) were tested by multiplex real-time RT-PCR. Mycobacterial liquid culture and Xpert MTB/RIF was performed on 2 induced sputa obtained from each participant. Children were categorised as definite-TB (culture or qPCR [Xpert MTB/RIF] confirmed), unlikely-TB (improvement of symptoms without TB treatment on follow-up) and unconfirmed-TB (all other children).

Results: Amongst 214 children with a median age of 36 months (interquartile range, [IQR] 19-66 months), 34 (16\%) had definite-TB, 86 (40 \%) had unconfirmed-TB and 94 (44 \%) were classified as unlikely-TB. Moraxella catarrhalis (64\%), Streptococcus pneumoniae (42\%), Haemophilus influenzae spp (29\%) and Staphylococcus aureus (22\%) were the most common bacteria detected in NP samples. Other bacteria detected included Mycoplasma pneumoniae (9\%), Bordetella pertussis (7\%) and Chlamydophila pneumoniae (4\%). The most common viruses detected included metapneumovirus (19\%), rhinovirus (15\%), influenza virus C (9\%), adenovirus (7\%), cytomegalovirus (7\%) and coronavirus $\mathrm{O} 43$ (5.6\%). Both bacteria and viruses were detected in 73, 55 and $56 \%$ of the definite, unconfirmed and unlikely-TB groups, respectively. There were no significant differences in the distribution of respiratory microbes between children with and without TB. Using quadratic discriminant analysis, human metapneumovirus, C. pneumoniae, coronavirus 043, influenza virus C virus, rhinovirus and cytomegalovirus best discriminated children with definite-TB from the other groups of children.

Conclusions: A broad range of potential respiratory pathogens was detected in children with suspected TB. There was no clear association between TB categorisation and detection of a specific pathogen. Further work is needed to explore potential pathogen interactions and their role in the pathogenesis of PTB.
\end{abstract}

Keywords: Infection, Nasopharynx, Microbiota, Mycobacterium tuberculosis, Tuberculosis, Respiratory microbes

\footnotetext{
* Correspondence: felix.dube@uct.ac.za

'Division of Medical Microbiology, Department of Pathology, Faculty of

Health Sciences, University of Cape Town, Cape Town, South Africa

Full list of author information is available at the end of the article
} 


\section{Background}

Lower respiratory tract infection (LRTI) is a leading cause of mortality and morbidity in children under five years, accounting for approximately 1 million deaths in 2013 globally [1]. Pulmonary tuberculosis (PTB) is an important cause of LRTI and may present as acute or chronic disease [2]. TB is increasingly recognised as a primary cause or as a comorbid condition in children hospitalized with pneumonia, particularly in settings endemic for human immunodeficiency virus (HIV) and TB [3].

Diagnosis of PTB in children is largely made based on clinical and radiological features, which may be nonspecific. Therefore PTB cannot be easily differentiated from other causes of acute or chronic LRTI [4]. Recent studies have reported detection of polymicrobial infections in children with LRTI [5]. Further, PTB has increasingly been reported in children presenting with acute pneumonia; culture confirmed TB was reported in $8 \%$ of South African children hospitalized with acute pneumonia with no difference by HIV status [6]. A recent meta-analysis confirmed Mycobacterium tuberculosis in $7.5 \%$ of childhood pneumonia cases in TB endemic areas [3].

There are limited published data on the role of other respiratory pathogens amongst patients suspected to have TB [7]. In Africa, only one study, conducted in Botswana, has addressed this question amongst adult PTB suspects. This study reported microbiologically confirmed TB in 118/229 (52 \%); Mycoplasma pneumoniae infection in 36/218 (17 \%) and Pneumocystis jirovecii infection in 4/141 (3\%) of PTB suspects [7]. Co-infection with two or more pathogens was documented in $25 \%$ of patients [7].

In our studies of South African children hospitalised with suspected PTB, $16 \%$ of children hospitalised with suspected PTB had microbiologically-confirmed PTB, $48 \%$ were classified as unconfirmed-PTB and $37 \%$ were classified as unlikely-PTB (children in whom TB was excluded and who improved in the absence of TB therapy) [8]. Approximately half of the children were treated for PTB, including all children with definite PTB and most with unconfirmed PTB [8]. These data raise several questions around potential co-infections including the extent to which these may contribute to disease or severity in PTB, the aetiology of LRTI amongst children with symptoms suggestive PTB and the role of other potential respiratory pathogens in those without TB.

We have recently shown [5] that specific pathogens (including Bordetella pertussis, influenza virus, respiratory syncyntial virus [RSV], adenovirus, parainfluenzavirus, bocavirus) are detected significantly more frequently from the nasopharynx (NP) of children with pneumonia than age-matched controls, and so are likely to play an important role in pneumonia aetiology. We therefore conducted a preliminary study to investigate the diversity of potential respiratory pathogens in the NP of children hospitalised with suspected PTB.

\section{Methods}

\section{Study population and TB testing}

The population studied in this study has been previously described [8]. Briefly, we enrolled children under 15 years of age suspected of PTB presenting (between July 2011 and May 2012) at Red Cross War Memorial Children's Hospital $(\mathrm{RCH})$, a specialist referral paediatric hospital in Cape Town, South Africa. Verbal assent was obtained from children older than seven (7) years of age and informed consent was obtained from a parent or legal guardian for all children.

Suspected TB was defined as having history of a cough and one of the following: i) a household contact with TB within the previous three months, ii) loss of weight or failure to gain weight in the previous three months, iii) a positive tuberculin skin test (TST) to purified protein derivative (PPD; 2TU, PPD RT23, Staten Serum Institute, Denmark, Copenhagen), or iv) a chest radiograph suggestive of PTB. A positive skin test was defined as $5 \mathrm{~mm}$ or more of transverse induration in children with HIV infection or $10 \mathrm{~mm}$ or more in children without HIV infection [9]. Children were excluded if the child was on TB treatment or TB prophylaxis for more than $72 \mathrm{~h}$, and if they could not be followed-up (not resident in Cape Town). All patients with laboratory confirmed $\mathrm{TB}$ and those diagnosed with TB based on clinical and radiological criteria were referred for TB therapy at a local clinic in accordance with South African National Guidelines [10]. Children were followed up at 1 and 3 months to assess response to therapy or improvement without TB treatment. All children received care in the public health system that includes free expanded programme on immunisation (EPI) for diphtheria, pertussis (whooping cough), and tetanus (DPT), Haemophilus influenza type b, and Streptococcus pneumoniae (7-valent pneumococcal conjugate vaccine [PCV] from 2009, replaced with 13-valent PCV in 2011).

\section{Sample collection}

Two paired induced sputa and a nasopharyngeal (NP) swab specimens were collected from each child and transported to the laboratory within $2 \mathrm{~h}$ of collection. NP swabs were obtained before sputum induction using nylon flocked swabs (Copan Italia, Brescia, Italy) by trained study staff [11]. Each NP swab was immediately placed into $1.5 \mathrm{ml}$ PrimeStore ${ }^{\oplus}$ transport and stabilization medium (PrimeStore ${ }^{\bullet}$ MTM, Longhorn Vaccines and Diagnostics, San Antonio, TX) and stored at $-80{ }^{\circ} \mathrm{C}$ within $2 \mathrm{~h}$ of collection until further batch processing. Samples 
were randomly selected from a convenience subset of 214 children, over a 1-year period for testing of NP specimens for other microbes.

As the volume obtained on induced sputum specimens was small, the entire specimen was required for detection of $M$ tuberculosis, for optimal management and as this study was nested within a broader study investigating better diagnostics for TB in children [11]. We did not want to compromise this primary aim and only NP samples were available for study of other respiratory pathogens.

\section{Diagnosis of PTB}

Induced sputum specimens were submitted to the National Health Laboratory Services (NHLS) Medical Microbiology Laboratory at Groote Schuur Hospital (Cape Town, South Africa) for mycobacterial liquid culture (BACTEC MGIT, Becton Dickinson Microbiology Systems, Cockeysville, MD) and nucleic acid amplification testing (Xpert MTB/RIF, Cepheid, Sunnyvale, CA).

Children were categorised as 'definite-TB' (i.e. culture or Xpert MTB/RIF positive for M. tuberculosis), 'unlikely TB' (i.e. no clinical diagnosis of TB with improvement on follow-up without TB treatment) and 'unconfirmed TB' (all others) [12].

\section{Multiplex PCR testing of nasopharyngeal samples}

$\mathrm{NP}$ swabs were thawed at room temperature $\left(22^{\circ} \mathrm{C}\right)$ and vortexed for $15 \mathrm{~s}$. Thereafter, $400 \mu \mathrm{l}$ of each sample was transferred to a ZR BashingBeadsTM Lysis tube (Zymo Research Corp., Irvine, CA) and subjected to mechanical lysis on a Tissuelyzer LT (Qiagen, Hilden, Germany) [13]. The lysed samples were then centrifuged at $10,000 \times \mathrm{g}$ for $1 \mathrm{~min}$ to pellet all cellular debris. Aliquots of $250 \mu \mathrm{l}$ of the supernatant were transferred to a $2 \mathrm{ml}$ sterile tube (Sarstedt, Nümbrecht, Germany) and $4 \mu \mathrm{l}$ of an exogenous internal control (Equine arteritis virus) was added to each sample prior to automated total nucleic acid extraction on the QIAsymphony SP instrument using the QIAsymphony Virus/Bacteria mini kit (Qiagen, Hilden, Germany). Total nucleic acid was eluted in $60 \mu \mathrm{l}$ elution buffer and stored at $-80{ }^{\circ} \mathrm{C}$ until further processing.

Nucleic acid amplification was performed using the FTD Resp33 kit according to the manufacturer's instructions (Fast-track Diagnostics, Luxembourg). The assay comprises eight multiplex real-time PCR reactions for the detection of nucleic acid targets (Additional file 1: Table S1).

Results were interpreted according to manufacturer's instructions using the FTD resp33 Analyser, an in-house JAVA based program (available at http://www.gematics. com/analyser.html).

\section{Statistical analysis}

Exploratory statistics were performed using STATA software (Stata Corporation, College Station, TX), whilst the openly available statistical environment $\mathrm{R}$, version 3.1.1 [14] was used for more detailed analyses. Pearson's Chisquared test was used to compare the occurrence of each microbe between children with definite TB and unlikely TB with Yates' continuity correction. Permutation tests were used to determine which microbe pairs were statistically concurrent. Briefly, for each pair of microbes, $X$ and $Y$, the observed number of concurrences $(m)$ was counted. The null hypothesis was that there was no relationship between microbes $X$ and $Y$ and that the co-occurrences were purely random. The null hypothesis was tested by generating random permutations of the occurrences of $X$ and $Y$. The number of concurrences under these random conditions was then counted, $m_{1}$. By repeating the permutation process 10000 times, 10 000 random concurrences were obtained as follows: $m_{1}$, $m_{2}, \ldots, m_{10000}$. The shape of the null-distribution, the distribution of co-occurrence counts under purely random conditions, was estimated from the 10000 observed values of the permutation test. The achieved significance level (ASL) was computed as the number of permuted co-occurrences that were equal to or greater than the observed number of concurrence (tail probability under the null-distribution). This can be interpreted as a nonparametric $p$-value [15].

Linear Discriminant Analysis (LDA) and Quadratic Discriminant Analysis (QDA) [16] were used to optimally discriminate respiratory microbes occurring in relation to TB status. The LDA is visually represented in a Canonical Variate Analysis (CVA) biplot and the QDA in a QDA biplot [16]. The visualizations provide information on how the different TB groups overlap and to what extent the detection of microbes differs between the groups. For all the tests, a $p$-value less than 0.05 was used as the limit of statistical significance.

\section{Ethical considerations}

The Human Research Ethics Committee (HREC 045/ 2008) of the Faculty of Health Sciences, University of Cape Town, South Africa approved this study.

\section{Results}

Table 1 summarises the baseline characteristics of the 214 children included in this study. The median age of the study participants was 36 months (interquartile range, [IQR] 19-66 months). Children with definite-TB were older $(p=0.003)$ than children with unconfirmed TB or unlikely TB. Thirty-four (16\%) of the 214 children had culture confirmed TB, while 86/214 (40\%) had unconfirmed-TB and 94/214 (44\%) were categorised as unlikely TB. Only $13 \%(27 / 214)$ of children were 
Table 1 Baseline characteristics of the children in the study by TB category

\begin{tabular}{|c|c|c|c|c|c|}
\hline Characteristics & Total $(n=214)$ & Definite-TB $(n=34)$ & Unconfirmed-TB $(n=86)$ & Unlikely-TB $(n=94)$ & $p$-value \\
\hline Age in months, median (IQR) & $36(19-66)$ & $56(23-109)$ & $34(15-63)$ & $32(17-63)$ & 0.003 \\
\hline Sex: female, n (\%) & $109(51)$ & $14(41)$ & $45(52)$ & $50(53)$ & 0.03 \\
\hline HIV infection, n (\%) & $27(13)$ & $7(21)$ & $13(15)$ & $7(7)$ & 0.14 \\
\hline \multicolumn{6}{|l|}{ HIV WHO clinical staging, n (\%) } \\
\hline Stage 1 & 0 & 0 & 0 & 0 & - \\
\hline Stage 2 & 0 & 0 & 0 & 0 & - \\
\hline Stage 3 & $21(10)$ & $5(15)$ & $10(12)$ & $6(6)$ & 0.36 \\
\hline Stage 4 & $6(3)$ & $2(6)$ & $3(35)$ & $1(1)$ & 0.48 \\
\hline Smoking: yes, n (\%) & $29(14)$ & $5(15)$ & $12(14)$ & $12(13)$ & 0.56 \\
\hline Night sweats: yes, n (\%) & $118(55)$ & $15(44)$ & $57(66)$ & $46(49)$ & 0.61 \\
\hline Fever: yes, n (\%) & $102(48)$ & $13(38)$ & $52(60)$ & $37(39)$ & 0.19 \\
\hline Malaise: yes, n (\%) & $62(29)$ & $14(41)$ & $30(35)$ & $18(19)$ & 0.88 \\
\hline Cough: yes, n (\%) & $165(77)$ & $27(79)$ & $67(78)$ & $71(76)$ & 0.61 \\
\hline Appetite loss: yes, n (\%) & $110(51)$ & $18(53)$ & $43(50)$ & $48(51)$ & 0.69 \\
\hline Weight loss: yes, n (\%) & $133(62)$ & $23(68)$ & $63(73)$ & $47(50)$ & 0.07 \\
\hline Vomiting: yes, n (\%) & $43(20)$ & $7(21)$ & $19(22)$ & $17(18)$ & 0.73 \\
\hline BMI, median (IQR) & $16(15-18)$ & $15(14-17)$ & $15(14-17)$ & $16(15-18)$ & 0.26 \\
\hline Hypoxia, median (IQR) & $99(97-100)$ & 98 (97-99) & $99(97-100)$ & $100(97-100)$ & 0.63 \\
\hline Chest in drawing: yes, n (\%) & $20(9)$ & $5(15)$ & $10(12)$ & $5(5)$ & 0.08 \\
\hline Respiratory rate, median (IQR) & $34(31-40)$ & $35(30-40)$ & $35(30-40)$ & $34(32-40)$ & 0.68 \\
\hline
\end{tabular}

HIV-infected, with similar HIV prevalence by TB category. Immunization records were available for 162 out of $214(76 \%)$ children included in this study. Among children for whom data on immunization profile was available, the vaccination status was up to date in $78 \%$ of children, while among the remaining $22 \%(36 / 162)$, at least one scheduled immunization was missing. None of the risk factors considered in Table 1 was associated with the occurrence of any microbes pertussis even after adjusting for potential confounders (Additional file 1: Table S3).

\section{Microbes detected in nasopharyngeal samples}

Nucleic acid of at least one of the 33 targeted respiratory microbes was detected in $97 \%$ of 214 NP specimens. The most common bacteria detected were Moraxella catarrhalis (64 \%), S. pneumoniae (42\%), H. influenzae spp (29\%) and Staphylococcus aureus (22\%) (Table 2). M. pneumoniae (9\%), B. pertussis (7\%) or C. pneumoniae (4.\%) were detected less frequently. The most frequently detected viral targets were human metapneumovirus (hMPV) (19\%), rhinovirus (15\%), influenza C virus (9\%), adenovirus (7\%), cytomegalovirus (7\%) and coronavirus O43 (5.6 \%) (Table 2).

Seasonal patterns were observed for hMPV, rhinovirus, enterovirus and influenza viruses with peak prevalence in late winter (August) and spring (November).
In addition, a seasonal pattern was detected for tuberculosis (Additional file 1: Figure S1). No distinct seasonal patterns were observed for other microbes.

A single bacterial target was detected in 39/214 (18 \%) of samples tested. Two bacterial targets were detected in 53/214 (25\%), and three bacterial targets detected in 111/214 (52 \%). A single viral target was detected in 71/214 (33\%) of samples, two viral targets in $47 / 214$ (22\%) and three or more viral targets in 10/214 (5 \%) specimens. Bacteria alone were detected in 86/214 (40\%) of samples, viruses alone were detected in 11/214 (5\%) of samples and both viruses and bacteria in 117/214 (55 \%) of samples. A detailed overview of all possible pairs of co-occuring respiratory microbes, irrespective of $\mathrm{TB}$ category (Additional file 1: Table S2). These co-occurences were further tested for significance (Table 3). Significant bacterial-bacterial associations included interactions between $M$. catarrhalis and each of M. pneumoniae, $S$. pneumoniae and $H$. influenza spp. Viral-viral associations included: bocavirus and influenza A virus, parainfluenza 1 virus and coronavirus NL63 as well as hMPV and enterovirus. Significant viral - bacterial associations were common particularly between $H$. influenza (type b or non-type b) and a range of viruses, including enteroviruses, hMPV, influenza $\mathrm{C}$, influenza $\mathrm{A}$ and cytomegalovirus. 
Table 2 Respiratory microbes detected from children presenting with suspected pulmonary tuberculosis ${ }^{\mathrm{a}}$

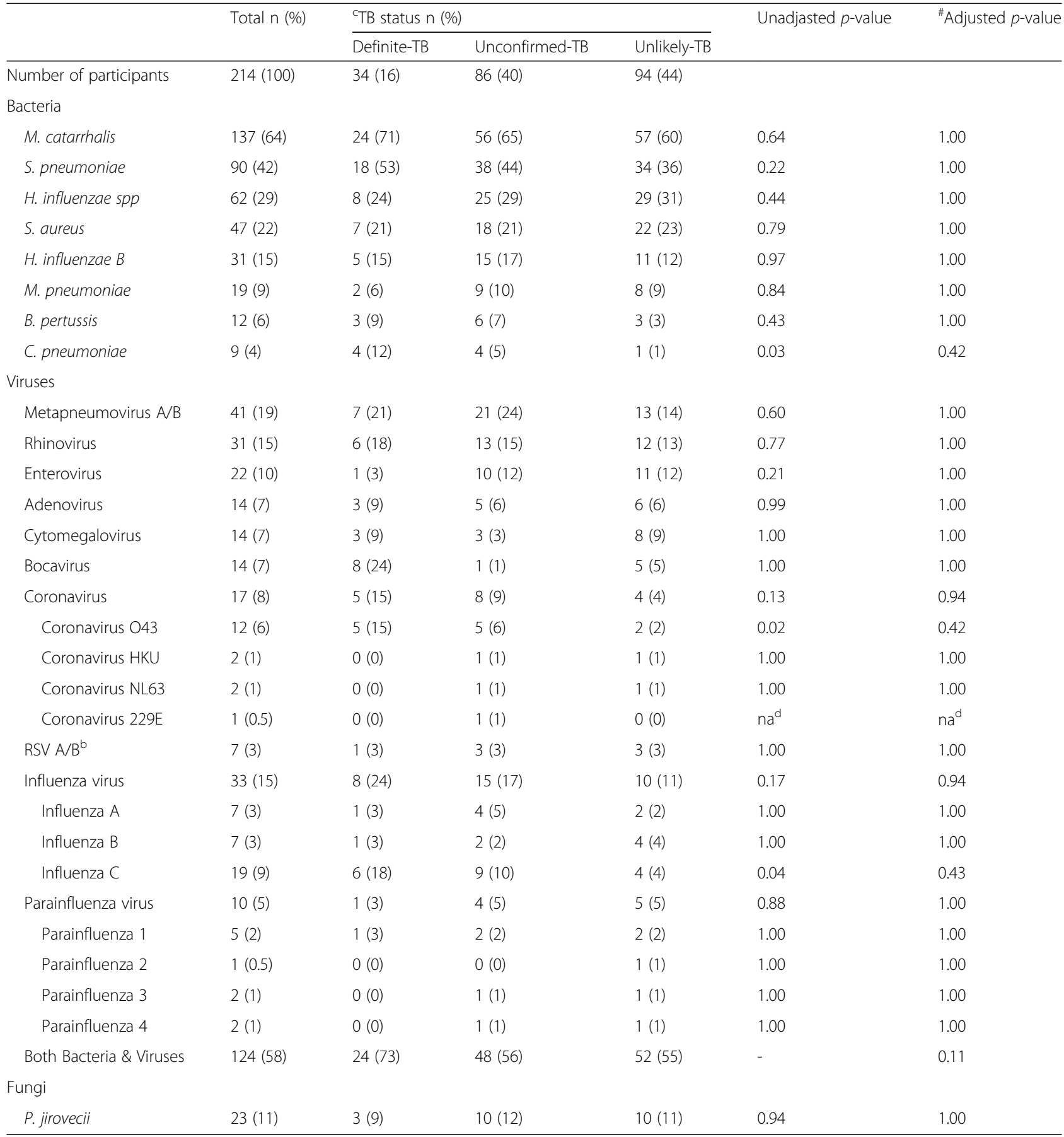

${ }^{a}$ Arranged in order of decreasing colonisation or detection rates

${ }^{b}$ RSV A/B = Respiratory Syncyntial virus A and B

"The adjusted $p$-value compensates for multiple comparisons. The $p$-values only compare the presence of each microbe between the definite-TB and unlikely-TB groups. The true proportion of TB in the unconfirmed-TB is unknown hence not included in comparison

${ }^{\mathrm{C}} \mathrm{TB}=$ Microbiological confirmation of pulmonary Tuberculosis

$\mathrm{na}^{\mathrm{d}}=$ Non of the children had Coronavirus 229 present, except in the unconfirmed-TB category which is excluded

\section{Microbial target detection by TB category}

Both bacterial and viral targets were detected in 24/34 (71 \%), 52/94 (55\%) and 48/86 (56 \%) of NP specimens from children with definite-TB, unconfirmed-TB and
unlikely-TB groups, respectively. There were no significant differences in the distribution of individual pathogens (Table 2) by TB category. However, visual inspection of the CVA biplot for graphical visualisation 
Table 3 Summary of significance level for all paired microbe co-occurrences ${ }^{c}$

\begin{tabular}{|c|c|c|c|c|c|c|c|c|c|c|c|c|c|}
\hline & $\begin{array}{l}\text { Influenza } \\
\text { A }\end{array}$ & Rhinovirus & $\begin{array}{l}\text { Influenza } \\
\text { B }\end{array}$ & $\begin{array}{l}\text { Parainfuenza } \\
3\end{array}$ & $\begin{array}{l}\text { Parainfuenza } \\
2\end{array}$ & $\begin{array}{l}\text { Parainfuenza } \\
4\end{array}$ & $\begin{array}{l}\text { Coronavirus } \\
29\end{array}$ & $\begin{array}{l}\text { Coronavirus } \\
63\end{array}$ & $\begin{array}{l}\text { Coronavirus } \\
43\end{array}$ & $\begin{array}{l}\text { Coronavirus } \\
\text { HK }\end{array}$ & RSV $A / B^{a}$ & Cytomegalovirus & Adenovirus \\
\hline Rhinovirus & 1 & & & & & & & & & & & & \\
\hline Influenza B & 1 & 1 & & & & & & & & & & & \\
\hline Parainfuenza 3 & 1 & 0.27 & 1 & & & & & & & & & & \\
\hline Parainfuenza 2 & 1 & 0.14 & 1 & 1 & & & & & & & & & \\
\hline Parainfuenza 4 & 1 & 0.27 & 1 & 1 & 1 & & & & & & & & \\
\hline Coronavirus 229 & 1 & 1 & 1 & 1 & 1 & 1 & & & & & & & \\
\hline Coronavirus 63 & 1 & 1 & 1 & 1 & 1 & 1 & 1 & & & & & & \\
\hline Coronavirus 43 & 1 & 0.56 & 0.33 & 1 & 1 & 1 & 1 & 1 & & & & & \\
\hline Coronavirus & 0.07 & 1 & 1 & 1 & 1 & 1 & 1 & 1 & 1 & & & & \\
\hline $\mathrm{RSV} A / \mathrm{B}^{\mathrm{a}}$ & 1 & 0.66 & 1 & 1 & 1 & 1 & 1 & 1 & 1 & 1 & & & \\
\hline Cytomegalovirus & 0.07 & 1 & 1 & 1 & 1 & 1 & 0.07 & 1 & 0.57 & 0.12 & 1 & & \\
\hline Adenovirus & 0.38 & 0.90 & 1 & 1 & 1 & 1 & 1 & 1 & 0.56 & 0.13 & 1 & 0.23 & \\
\hline Enterovirus & 1 & 0.08 & 0.54 & 1 & 1 & 0.20 & 1 & 1 & 1 & 1 & 1 & 0.80 & 0.16 \\
\hline Parainfluenza 1 & 1 & 1 & 1 & 1 & 1 & 1 & 1 & 0.04 & 0.25 & 1 & 1 & 1 & 1 \\
\hline $\mathrm{HmPV} A / B^{b}$ & 0.78 & 0.57 & 0.78 & 0.3 & 1 & 1 & 1 & 1 & 0.41 & 1 & 1 & 0.27 & 0.79 \\
\hline M. pneumoniae & 0.48 & 0.29 & 1 & 0.01 & 1 & 0.17 & 1 & 1 & 0.68 & 1 & 0.12 & 1 & 0.36 \\
\hline Bocavirus & 0.02 & 0.33 & 1 & 1 & 1 & 1 & 1 & 1 & 1 & 0.08 & 0.24 & 1 & 0.42 \\
\hline S. aureus & 1 & 0.72 & 0.83 & 1 & 1 & 0.39 & 1 & 1 & 0.79 & 1 & 0.82 & 0.37 & 0.97 \\
\hline C. pneumoniae & 1 & 0.76 & 1 & 1 & 1 & 1 & 1 & 1 & 1 & 1 & 0.26 & 0.47 & 0.46 \\
\hline S. pneumoniae & 0.11 & 0.09 & 0.62 & 1 & 1 & 0.67 & 1 & 1 & 0.19 & 0.67 & 0.98 & 0.18 & 0.77 \\
\hline H. influenzae B & 1 & 0.69 & 1 & 1 & 1 & 0.27 & 1 & 1 & 0.85 & 1 & 1 & 0.63 & 0.12 \\
\hline P. jirovecii & 0.55 & 0.44 & 0.55 & 1 & 1 & 1 & 1 & 0.21 & 0.38 & 0.01 & 0.55 & 0.46 & 0.81 \\
\hline M. cartarrhalis & 0.51 & 0.07 & 0.21 & 0.42 & 1 & 0.88 & 1 & 0.41 & 0.03 & 0.87 & 0.51 & 0.38 & 0.97 \\
\hline Influenza C & 1 & 0.79 & 1 & 1 & 1 & 1 & 1 & 1 & 0.30 & 1 & 0.12 & 1 & 0.75 \\
\hline H. influenzae spp & 0.02 & 0.57 & 0.32 & 0.49 & 1 & 1 & 1 & 1 & 0.47 & 0.50 & 0.91 & 0.00 & 0.39 \\
\hline B. pertussis & 1 & 0.54 & 0.33 & 1 & 1 & 1 & 1 & 1 & 0.51 & 1 & 1 & 1 & 1 \\
\hline
\end{tabular}

${ }^{a} \mathrm{RSV} A / B=$ Respiratory Syncyntial virus $\mathrm{A}$ and $\mathrm{B},{ }^{\mathrm{b}} \mathrm{HmPV} \mathrm{A} / \mathrm{B}=$ Human Metapneumovirus $\mathrm{A}$ and $\mathrm{B}$

'Each pair of microbe pairs were tested for concurrency and the table reports achieved significance levels (interpreted similar to a $p$-value) irrespective of the TB category. Significant concurrent relationships are highlighted in bold 
Table 3 Summary of significance level for all paired microbe co-occurrences ${ }^{c}$ (Continued)

\begin{tabular}{|c|c|c|c|c|c|c|c|c|c|c|c|c|c|}
\hline & Enterovirus & Parainfuenza 1 & $\mathrm{HmPV} A / \mathrm{B}^{\mathrm{b}}$ & M. pneumoniae & Bocavirus & S. aureus & C. pneumoniae & S. pneumoniae & H. influenzae B & P. jirovecii & M. cartarrhalis & Influenza C & $\begin{array}{l}\text { H. influenzae } \\
\text { spp }\end{array}$ \\
\hline \multicolumn{14}{|l|}{ Rhinovirus } \\
\hline \multicolumn{14}{|l|}{ Influenza B } \\
\hline \multicolumn{14}{|l|}{ Parainfuenza 3} \\
\hline \multicolumn{14}{|l|}{ Parainfuenza 2} \\
\hline \multicolumn{14}{|l|}{ Parainfuenza 4} \\
\hline \multicolumn{14}{|l|}{ Coronavirus 229} \\
\hline \multicolumn{14}{|l|}{ Coronavirus 63} \\
\hline \multicolumn{14}{|l|}{ Coronavirus 43} \\
\hline \multicolumn{14}{|l|}{ Coronavirus } \\
\hline \multicolumn{14}{|l|}{$\mathrm{RSV} A / \mathrm{B}^{\mathrm{a}}$} \\
\hline \multicolumn{14}{|l|}{ Cytomegalovirus } \\
\hline \multicolumn{14}{|l|}{ Adenovirus } \\
\hline \multicolumn{14}{|l|}{ Enterovirus } \\
\hline Parainfluenza 1 & 1 & & & & & & & & & & & & \\
\hline $\mathrm{HmPV} A / B^{b}$ & 0.01 & 1 & & & & & & & & & & & \\
\hline M. pneumoniae & 0.31 & 1 & 0.51 & & & & & & & & & & \\
\hline Bocavirus & 0.58 & 1 & 0.82 & 1 & & & & & & & & & \\
\hline S. aureus & 0.08 & 1 & 0.26 & 0.83 & 0.25 & & & & & & & & \\
\hline C. pneumoniae & 0.63 & 0.19 & 1 & 0.58 & 0.29 & 0.89 & & & & & & & \\
\hline S. pneumoniae & 0.63 & 0.94 & 0.03 & 0.76 & 0.73 & 0.28 & 0.99 & & & & & & \\
\hline H. influenzae $B$ & 0.02 & 1 & 0.75 & 0.04 & 1 & 0.36 & 0.76 & 0.43 & & & & & \\
\hline P. jirovecii & 0.72 & 1 & 0.68 & 0.63 & 0.60 & 0.00 & 1 & 0.70 & 0.00 & & & & \\
\hline M. cartarrhalis & 0.05 & 0.95 & 0.06 & 0.01 & 1 & 0.81 & 0.94 & 0.00 & 0.71 & 0.72 & & & \\
\hline Influenza C & 0.61 & 0.06 & 0.91 & 0.02 & 0.53 & 1 & 0.19 & 1 & 0.00 & 0.63 & 0.45 & & \\
\hline H. influenzae spp & 0.92 & 0.82 & 0.02 & 0.69 & 0.73 & 0.99 & 0.52 & 0.01 & 0.86 & 0.71 & 0.03 & 1 & \\
\hline B. pertussis & 0.12 & 1 & 0.93 & 0.29 & 1 & 1 & 0.41 & 0.94 & 0.00 & 1 & 0.13 & 0.00 & 0.48 \\
\hline
\end{tabular}


of LDA for all TB categories (Fig. 1) suggests that C. pneumoniae, S. pneumoniae, M. catarrhalis, coronavirus O43, influenza virus $C$ virus, rhinovirus, parainfluenza virus 1 , and adenovirus formed the dominant microbial profile in definite-TB cases. In contrast, cytomegalovirus, influenza B, parainfluenza virus $2, \mathrm{RSV}$ A/B, S. aureus, $H$. influenzae $s p p$, and $P$. jirovecii associated with the unlikely-TB group. When the unconfirmed TB group is excluded from the LDA analysis, the LDA biplot is reduced to a onedimensional plot (Additional file 1: Figure S2). In this case, the presence of rhinovirus, coronavirus 043, adenovirus, parainfluenza 1, hMPV, bocavirus, C. pneumoniae, $S$. pneumoniae, $H$. influenzae type $b, M$. catarrhalis, influenza virus $C$ virus, and $B$. pertussis best discriminated cases with definite-TB from those with unlikely TB.

Quadratic Discriminent Analysis did not identify any significant association between definite-TB and unlikely-
TB groups. However, visual inspection of the QDA biplot (Fig. 2) showed that hMPV, coronavirus 043, influenza $C$ virus, rhinovirus, cytomegalovirus and $C$. pneumoniae formed the dominant microbial profile associated with definite-TB cases. In contrast, M. pneumoniae, $H$. influenzae, $P$. jirovecii, enterovirus, influenza $\mathrm{B}$ virus and RSV A/B were associated with the unlikely-TB category.

\section{Discussion}

This is the first comprehensive detailed study of the diversity of respiratory microbes detected in children presenting with suspected PTB in a TB endemic setting and showed that multiple potential pathogens are present in th nasopharynx of such children.

The FTD33 multiplex real-time PCR detected at least one of the 33 microbial targets in $97 \%$ of NP swabs

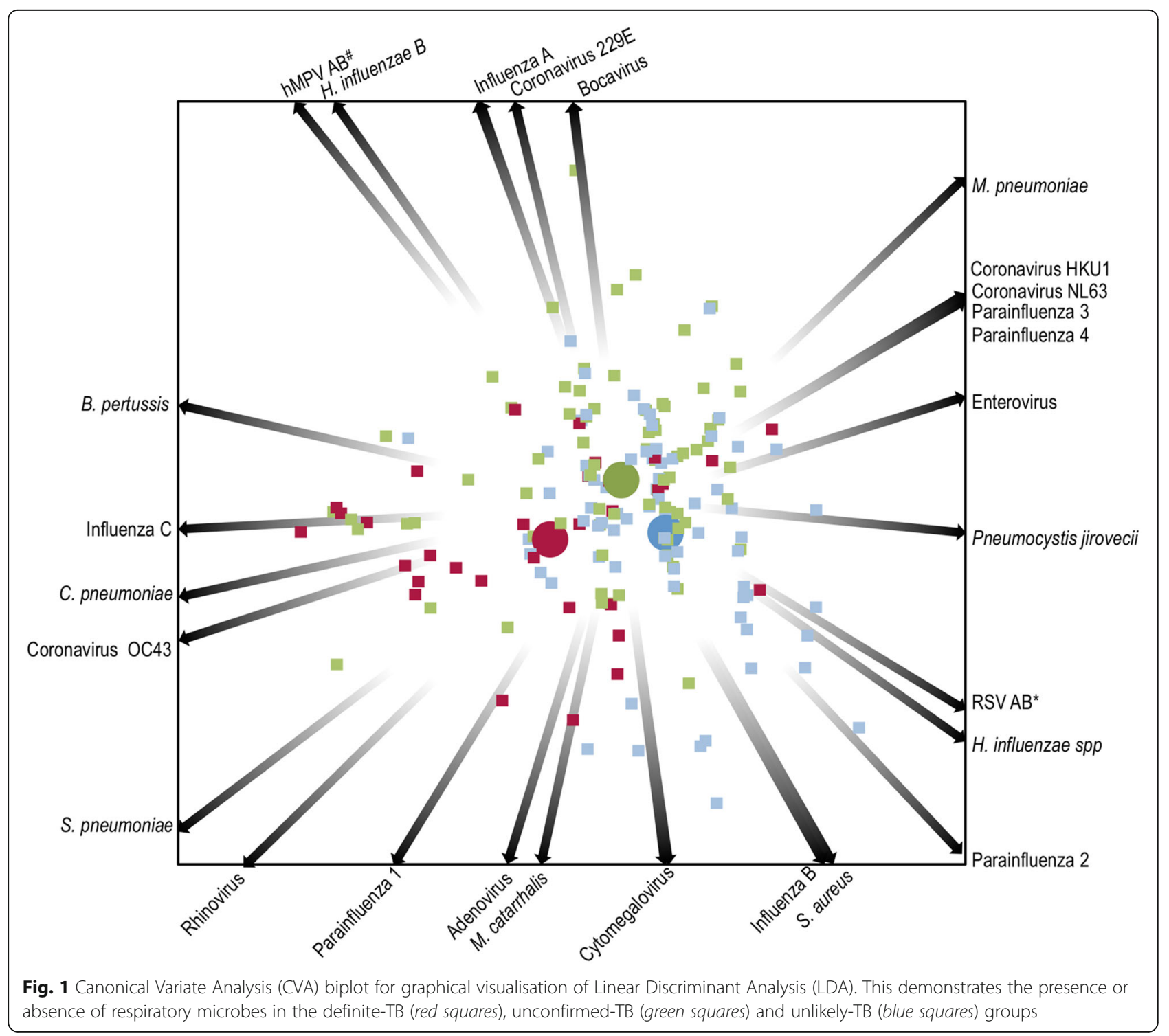




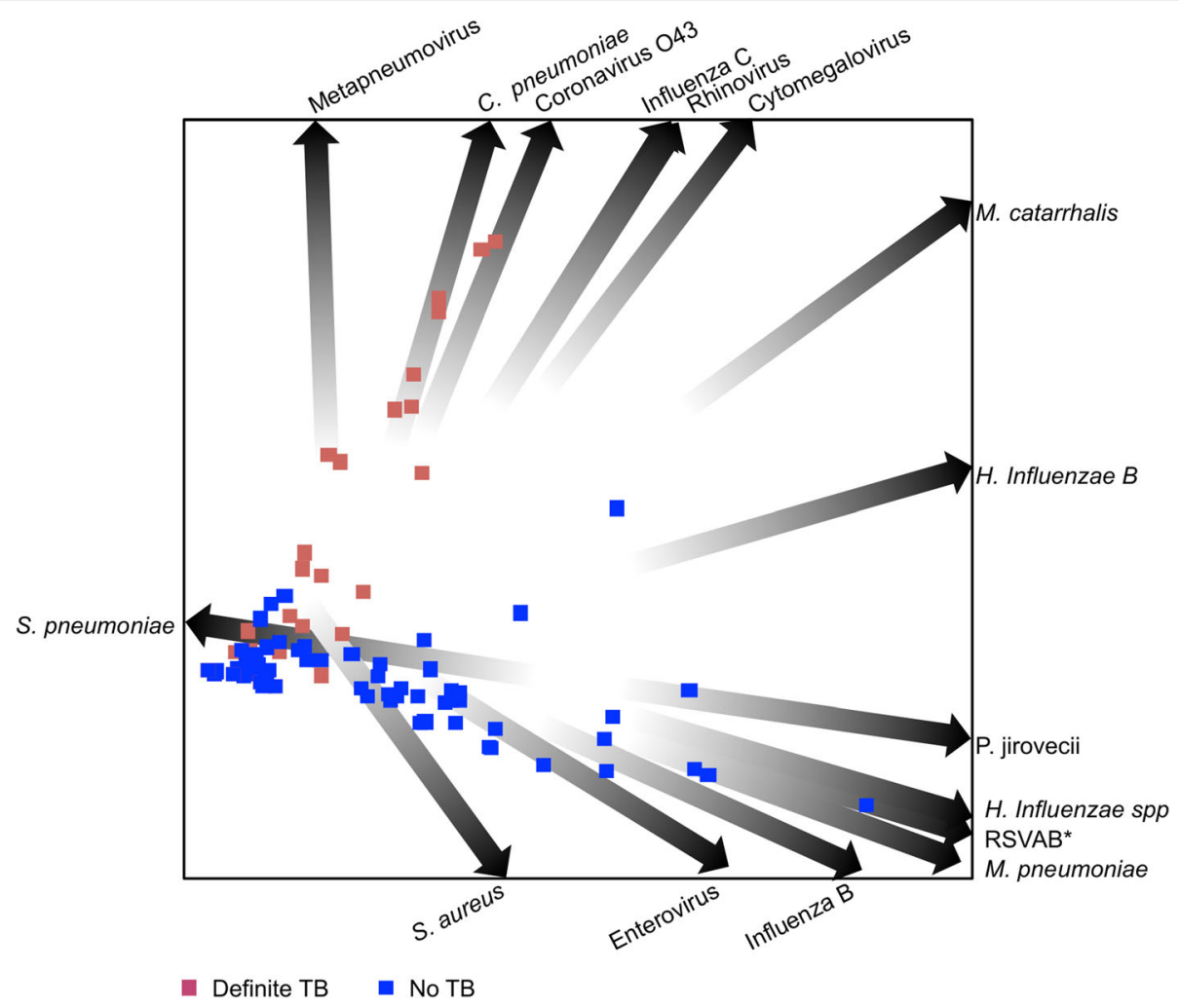

Fig. 2 Quadratic Discriminant Analysis (QDA) biplot for graphical visualisation of microbe classification [16]. Clustering of respiratory microbes in the definite-TB (red squares) and unlikely-TB (blue squares) groups is shown. Arrows indicate the presence of the microbes. *RSVAB $=$ Respiratory Syncytial virus $A$ and $B$

from children suspected of PTB. Detection of multiple bacterial and viral targets was common. Bacterial species frequently found as commensals in the nasopharynx were most commonly detected $[17,18]$. They include $M$. catarrhalis (64\%), S. pneumoniae (42\%), H. influenzae spp (29\%), and S. aureus (22\%). In addition, potential pathogenic organisms were detected in the nasopharynx including RSV, C. pneumoniae and B. pertussis.

The prevalence of $B$. pertussis was $6 \%$ in our study. Similar detection rates (1-9 \%) were reported in other South African settings in children with LRTI including at our study site, 10-20 years post transition from whole-cell vaccines to acellular vaccines (South African infants are vaccinated with DTaP-IPV/HIB; Pentaxime ${ }^{\circ}$, Sanofi Pasteur) [19].

The prevalence of some clinically relevant viral targets (RSV, bocavirus and adenovirus) in this cohort is lower than that previously reported in children with LRTI [20-22]. The observed differences may be explained by our enrolment criteria which targeted symptoms suggestive of PTB. However, viral PCR positivity of hMPV, enterovirus and influenza virus is similar to a recent case-control study that also showed their association with community acquired pneumonia [20]. As with bacteria, care needs to be taken with the interpretation of molecular detection of some viruses in NP specimens, since target nucleic acid may be detected for some time after resolution of symptoms, and from otherwise healthy children [23, 24].

In this study, some microbes showed no association with any of the TB categories. These included M. catarrhalis, and S. pneumoniae. A randomised controlled trial of the efficacy of PCV9 in South African children showed decreased rates of culture-confirmed and clinically diagnosed TB in PCV9 recipients hospitalised with LRTI compared with placebo recipients (relative risk reduction $43 \%$ ) [6]. This suggests that coinfection with $M$. tuberculosis and S. pneumoniae causes severe infection requiring hospitalization. Although common in our cohort, S. pneumoniae did not cluster together with the TB or unlikely-TB groups, however we measured NP colonization which is likely to be an inaccurate measure for the contribution of S. pneumoniae to LRTI.

We have recently shown [5] that specific pathogens (specifically B. pertussis, influenza virus, RSV, adenovirus, parainfluenzavirus, bocavirus) are detected significantly more frequently from the NP of children with pneumonia than age-matched controls. In this study we detected all of these organisms, irrespective of TBclassification, suggesting that these pathogens may play a role in the exacerbation of symptoms in children with 
TB as well as accounting for the respiratory illness of a subset of the children without TB.

Whilst we detected multiple significant co-occurrences between different microbes in this study, these require more detailed assessment in a larger group of children. For example, the co-occurrence between $M$. catarrhalis, $S$. pneumoniae and $H$. influenzae, may reflect age-specific colonization patterns as previously reported $[25,26]$. Other microbial co-occurrences, such as the association between $S$. aureus and $P$. jorovecii, were unexpected. We are currently conducting a larger, longitudinal study to better understand these co-occurences [27].

We were unable to identify significant associations between individual nasopharyngeal microbes and TB classification. Discriminant analysis identified that the presence of C. pneumoniae, hMPV, coronavirus O43, influenza $\mathrm{C}$ virus, rhinovirus and cytomegalovirus best discriminated children with definite TB. The significance of co-detection of these microbes in children with TB is unclear, and needs to be further assessed. One possibility is that the relative immune suppression or lung pathology associated with PTB may render the host susceptible to other respiratory infections, or alternatively, that intercurrent infection may be immunosuppressive, predisposing to an accelerated clinical course or likelihood of symptoms in children with PTB. Active TB is associated with suppression of cellular immune responses, which are critical for the control of intracellular infections [28] such as many of those associated with definite TB in this study. However, in this study, individual microbes were each only detected in small numbers of children which limits our ability to draw firm conclusions in this regard.

A recent South African study has shown an increased risk of death in adults with TB-influenza A virus coinfection (adjusted relative risk ratio [aRRR] 6.1) compared to TB infection alone [29]. In contrast, de Paus et al. did not find a correlation between the seroprevalence of influenza antibodies and the development of clinically active TB in an Indonesian cohort [30]. They did however show an association between elevated antibody titres against influenza A and the clinical stage of TB lung disease suggesting recent re-infection with influenza precedes clinical presentation with PTB [30].

A limitation of this study is the lack of a control group of children without lower respiratory symptoms. We are therefore unable to infer whether the pathogens detected played a role in the development or exacerbation of symptoms in this cohort. Further limitations include sampling of the nasopharynx rather than the lower respiratory tract, limiting the ability to infer causality for lung co-pathogens. Klebsiella pneumoniae, Legionella spp and Salmonella targets were excluded from analysis due to problems with assay specificity for these targets.

\section{Conclusion}

In conclusion, this study describes the detection of multiple respiratory microbes in the nasopharynx of children hospitalised with suspected PTB. Whilst there was no clear separation between the pathogens present in the airways of children with and without PTB, C. pneumoniae, $\mathrm{hMPV}$, coronavirus $\mathrm{O} 43$, influenza $\mathrm{C}$ virus, rhinovirus and cytomegalovirus formed the dominant microbial profile in children with PTB but this failed to reach statistical significance on testing of each individual microbe. In contrast, $P$. jirovecii, $H$. influenzae spp, RSV, M. pneumoniae, influenza $B$ virus and enteroviruses were more consistently detected in children without TB although not statistically significant. This pilot work may signal broader differences in the microbial ecology of the upper respiratory tract of these children, which warrants further study.

\section{Additional file}

Additional file 1: Figure S1. Seasonal distribution of viruses and bacteria. Figure S2. Canonical Variate Analysis (CVA) biplot depicting the spread of respiratory pathogens in the definite TB (red line) and not TB (blue line) groups only. Observations under each group are denoted by " + " signs and the median of each group by the red and blue ovals. Table S1. Target pathogens in the FTD respiratory pathogens 33 multiplex realtime PCR assay. Table S2. Summary of all paired pathogen co-occurrence counts *. Table S3. Risk factors associated with the occurrence of each microbes. (PDF $565 \mathrm{~kb}$ )

\begin{abstract}
Acknowledgements
We thank the National Health Laboratory Service diagnostic microbiology at Groote Schuur Hospital (Cape Town, South Africa) for the microbiological TB testing. We further wish to thank Lesley Workman, Charmaine Barthus, Widaad Zemanay, Layla Hendricks, Nchimunya Hapeela, Whitney Barnett and the rest of the study team for their help and technical assistance. We thank the Western Cape Health Department and the staff at Red Cross Children's Hospital for their support of the study.
\end{abstract}

\section{Funding}

This study was funded by the National Institutes of Health, USA (H3Africa Initiative 1U01Al110466-01A1 and 1R01HD058971-01) and the Medical Research Council of South Africa (Tuberculosis Collaborating Centre for Child Health). FSD is supported by the National Research Foundation of South Africa (UID99696) and further received funding from the University of Cape Town Faculty Research Committee (FRC) Postgraduate Publication Incentive. MK was supported by Carnegie Corporation of New York (USA) and now is supported by Wellcome Trust, UK (102429/Z/13/Z)

Availability of data and materials

Informations in our database is confidential, however, data used for the analysies is available upon request.

\section{Authors' contributions}

MPN, MK and HJZ conceptualised and supervised this study. MPN and HJZ obtained funding. FSD performed the experiments and analysed data with supervision from SL, who performed the Linear and Quadratic Discriminant Analysis (LDA and QDA). MK, MPN, HJZ, FJLR, SL and LAT contributed to experimental design, data analysis and manuscript preparation. All authors reviewed, contributed to, and approved the final manuscript.

Competing interests

The authors declare that they have no competing interests. 


\section{Consent for publication}

Not applicable.

\section{Ethics approval and consent to participate}

The Human Research Ethics Committee (HREC 045/2008) of the Faculty of Health Sciences, University of Cape Town, South Africa approved this study.

\section{Author details}

'Division of Medical Microbiology, Department of Pathology, Faculty of Health Sciences, University of Cape Town, Cape Town, South Africa. ${ }^{2}$ Faculty of Health Sciences, Institute for Infectious Diseases and Molecular Medicine, University of Cape Town, Cape Town, South Africa. ${ }^{3}$ Department of Statistical Sciences, Faculty of Science, University of Cape Town, Cape Town, South Africa. ${ }^{4}$ Department of Paediatrics and Child Health, Red Cross War Memorial Children's Hospital, University of Cape Town, Cape Town, South Africa. ${ }^{5}$ SAMRC Unit on Child and Adolescent Health, University of Cape Town, Cape Town, South Africa. ${ }^{6}$ National Health Laboratory Service, Groote Schuur Hospital, Cape Town, South Africa

Received: 29 January 2016 Accepted: 15 October 2016

Published online: 24 October 2016

\section{References}

1. Liu L, Oza S, Hogan D, Perin J, Rudan I, et al. Global, regional, and national causes of child mortality in 2000-13, with projections to inform post-2015 priorities: an updated systematic analysis. Lancet. 2015; 385:430-40. Available: http://www.ncbi.nlm.nih.gov/pubmed/25280870. Accessed 3 Oct 2014.

2. Lin PL, Flynn JL. Understanding latent tuberculosis: a moving target. J Immunol. 2010;185:15-22. Available: http://www.pubmedcentral.nih.gov/ articlerender.fcgi?artid=3311959\&tool=pmcentrez\&rendertype=abstract. Accessed 4 Aug 2014.

3. Oliwa JN, Karumbi JM, Marais BJ, Madhi SA, Graham SM. Tuberculosis as a cause or comorbidity of childhood pneumonia in tuberculosis-endemic areas: a systematic review. Lancet Respir Med. 2015;3:235-43. Available: http://www.ncbi.nlm.nih.gov/pubmed/25648115. Accessed 23 Apr 2015.

4. Connell TG, Zar HJ, Nicol MP. Advances in the diagnosis of pulmonary tuberculosis in HIV-infected and HIV-uninfected children. J Infect Dis. 2011;204(Suppl):S1151-8. Available: http://www.pubmedcentral.nih.gov/ articlerender.fcgi?artid=3192545\&tool=pmcentrez\&rendertype=abstract. Accessed 7 Feb 2015.

5. Zar HJ, Barnett W, Stadler A, Gardner-Lubbe S, Myer L, et al. Aetiology of childhood pneumonia in a well vaccinated South African birth cohort: a nested case-control study of the Drakenstein Child Health Study. Lancet Respir Med. 2016;4:463-72. Available: http://www.ncbi.nlm.nih.gov/pubmed/ 27117547. Accessed 22 Aug 2016.

6. Moore DP, Klugman KP, Madhi SA. Role of Streptococcus pneumoniae in hospitalization for acute community-acquired pneumonia associated with culture-confirmed Mycobacterium tuberculosis in children: a pneumococcal conjugate vaccine probe study. Pediatr Infect Dis J. 2010;29:1099-104. Available: http://www.ncbi.nlm.nih.gov/pubmed/21155174. Accessed 23 Feb 2015.

7. Lockman S, Hone N, Kenyon TA, Mwasekaga M, Villauthapillai M, et al. Etiology of pulmonary infections in predominantly HIV-infected adults with suspected tuberculosis, Botswana. Int J Tuberc Lung Dis. 2003;7:714-23. Available: http://www.ncbi.nlm.nih.gov/pubmed/12921146. Accessed 23 Feb 2015

8. Nicol MP, Workman L, Isaacs W, Munro J, Black F, et al. Accuracy of the Xpert MTB/RIF test for the diagnosis of pulmonary tuberculosis in children admitted to hospital in Cape Town, South Africa: a descriptive study. Lancet Infect Dis. 2011;11:819-24. Available: http://www.ncbi.nlm.nih.gov/pubmed/ 21764384. Accessed 3 Aug 2014.

9. Nicol MP, Allen V, Workman L, Isaacs W, Munro J, et al. Urine lipoarabinomannan testing for diagnosis of pulmonary tuberculosis in children: a prospective study. Lancet Glob Heal. 2014;2:e278-84. Available: http://www.pubmedcentral.nih.gov/articlerender.fcgi?artid=4012567\&tool= pmcentrez\&rendertype=abstract. Accessed 16 Feb 2015.

10. National Department of Health South Africa. National Tuberculosis Management Guidelines 2014. 2014; Available: https://www.google.gm/ webhp? sourceid=chrome-instant\&ion $=1 \&$ espv $=2 \&$ ie $=U T F-8 \# q=$ Available $\% 3 \mathrm{~A}$
+http\%3A\%2F\%2Fwww.sahivsoc.org\%2Fupload\%2Fdocuments\%2FNTCP_ Adult TB+Guidelines+27.5.2014.pdf. Accessed 3 Aug 2014.

11. Dube FS, Kaba M, Whittaker E, Zar HJ, Nicol MP. Detection of streptococcus pneumoniae from different types of nasopharyngeal swabs in children. PLoS One. 2013;8:e68097. Available: http://www.pubmedcentral.nih.gov/ articlerender.fcgi?artid=3694050\&tool=pmcentrez\&rendertype=abstract. Accessed 11 May 2014

12. Graham SM, Ahmed T, Amanullah F, Browning R, Cardenas V, et al. Evaluation of tuberculosis diagnostics in children: 1. Proposed clinical case definitions for classification of intrathoracic tuberculosis disease. Consensus from an expert panel. J Infect Dis. 2012;205(Suppl):S199-208. Available: http://www.pubmedcentral.nih.gov/articlerender.fcgi?artid=3334506\&tool= pmcentrez\&rendertype=abstract. Accessed 31 Mar 2016.

13. Claassen S, du Toit E, Kaba M, Moodley C, Zar HJ, et al. A comparison of the efficiency of five different commercial DNA extraction kits for extraction of DNA from faecal samples. J Microbiol Methods. 2013;94:103-10. Available: http://www.ncbi.nlm.nih.gov/pubmed/23684993. Accessed 9 Oct 2015.

14. R Core Team. R: A language and environment for statistical computing. 2014; Available: http://www.r-project.org/. Accessed 31 Mar 2014.

15. Efron B, Tibshirani R. An introduction to the bootstrap. New York: Chapman and Hall; 1993. Available: http://www.hms.harvard.edu/bss/neuro/bornlab/ nb204/statistics/bootstrap.pdf. Accessed 3 Mar 2014.

16. Gardner-Lubbe S, Dube FS. Visualisation of quadratic discriminant analysis and its application in exploration of microbial interactions. BioData Min. 2015;8:8. Available: http://www.biodatamining.org/content/8/1/8.

17. van den Bergh MR, Biesbroek G, Rossen JWA, de Steenhuijsen Piters WAA, Bosch AATM, et al. Associations between pathogens in the upper respiratory tract of young children: interplay between viruses and bacteria. PLoS One. 2012;7:e47711. Available: http://www.pubmedcentral.nih.gov/ articlerender.fcgi?artid=3474735\&tool=pmcentrez\&rendertype=abstract. Accessed 4 Nov 2014

18. Tenenbaum T, Franz A, Neuhausen N, Willems R, Brade J, et al. Clinical characteristics of children with lower respiratory tract infections are dependent on the carriage of specific pathogens in the nasopharynx. Eur J Clin Microbiol Infect Dis. 2012;31:3173-82. Available: http://www.ncbi.nlm. nih.gov/pubmed/22850740. Accessed 15 Dec 2014.

19. Muloiwa R, Dube FS, Nicol MP, Zar HJ, Hussey GD. Incidence and diagnosis of Pertussis in South African children hospitalized with lower respiratory tract infection. Pediatr Infect Dis J. 2016; Available: http://www.ncbi.nlm.nih. gov/pubmed/26967813. Accessed 29 Mar 2016.

20. Rhedin S, Lindstrand A, Hjelmgren A, Ryd-Rinder M, Öhrmalm L, et al. Respiratory viruses associated with community-acquired pneumonia in children: matched case-control study. Thorax. 2015;70:847-53. Available: http://www.ncbi.nlm.nih.gov/pubmed/26077969. Accessed 30 Mar 2016.

21. Tsolia MN, Psarras S, Bossios A, Audi H, Paldanius M, et al. Etiology of community-acquired pneumonia in hospitalized school-age children: evidence for high prevalence of viral infections. Clin Infect Dis. 2004;39:681-6. Available: http://cid.oxfordjournals.org/cgi/content/long/39/5/681. Accessed 30 Mar 2016.

22. Bezerra PGM, Britto MCA, Correia JB, Duarte Mdo C, Fonceca AM, et al. Viral and atypical bacterial detection in acute respiratory infection in children under five years. PLoS One. 2011;6:e18928. Available: http:// www.pubmedcentral.nih.gov/articlerender.fcgi?artid=3078930\&tool= pmcentrez\&rendertype=abstract. Accessed 4 Feb 2015.

23. Nunes MC, Kuschner Z, Rabede Z, Madimabe R, Van Niekerk N, et al. Clinical epidemiology of bocavirus, rhinovirus, two polyomaviruses and four coronaviruses in HIV-infected and HIV-uninfected South African children. PLoS One. 2014;9:e86448. Available: http://www.pubmedcentral.nih.gov/ articlerender.fcgi?artid=3911925\&tool=pmcentrez\&rendertype=abstract. Accessed 24 June 2015.

24. Jartti T, Jartti L, Peltola V, Waris M, Ruuskanen O. Identification of respiratory viruses in asymptomatic subjects: asymptomatic respiratory viral infections. Pediatr Infect Dis J. 2008;27:1 103-7. Available: http://www.ncbi.nlm.nih.gov/ pubmed/18978518. Accessed 11 Jan 2015.

25. García-Rodríguez JA, Fresnadillo Martínez MJ. Dynamics of nasopharyngeal colonization by potential respiratory pathogens. J Antimicrob Chemother. 2002; 59-73. Available: http://www.ncbi.nlm.nih.gov/pubmed/12556435. Accessed 26 Sept 2016.

26. Kwambana BA, Barer MR, Bottomley C, Adegbola RA, Antonio M. Early acquisition and high nasopharyngeal co-colonisation by Streptococcus pneumoniae and three respiratory pathogens amongst Gambian new-borns 
and infants. BMC Infect Dis. 2011;11:175. Available: http://www.pubmed central.nih.gov/articlerender.fcgi?artid=3129300\&tool=pmcentrez\& rendertype=abstract. Accessed 29 Jan 2015.

27. Zar HJ, Barnett W, Myer L, Stein DJ, Nicol MP. Investigating the early-life determinants of illness in Africa: the Drakenstein child health study. Thorax. 2014; Available: http://www.ncbi.nlm.nih.gov/pubmed/25228292. Accessed 3 Jan 2015.

28. Roberts T, Beyers N, Aguirre A, Walzl G. Immunosuppression during active tuberculosis is characterized by decreased interferon- gamma production and $\mathrm{CD} 25$ expression with elevated forkhead box P3, transforming growth factor- beta, and interleukin-4 mRNA levels. J Infect Dis. 2007;195:870-8. Available: http://www.ncbi.nlm.nih.gov/pubmed/17299718. Accessed 5 Sept 2016.

29. Walaza S, Tempia S, Dawood H, Variava E, Moyes J, et al. Influenza virus infection is associated with increased risk of death amongst patients hospitalized with confirmed pulmonary tuberculosis in South Africa, 2010;2011. BMC Infect Dis. 2015;15:26. Available: http://www. pubmedcentral.nih.gov/articlerender.fcgi?artid=4316613\&tool= pmcentrez\&rendertype=abstract. Accessed 28 Feb 2015.

30. de Paus RA, van Crevel R, van Beek R, Sahiratmadja E, Alisjahbana B, et al. The influence of influenza virus infections on the development of tuberculosis. Tuberculosis (Edinb). 2013;93:338-42. Available: http://www. ncbi.nlm.nih.gov/pubmed/23474302. Accessed 28 Feb 2015.

\section{Submit your next manuscript to BioMed Central and we will help you at every step:}

- We accept pre-submission inquiries

- Our selector tool helps you to find the most relevant journal

- We provide round the clock customer support

- Convenient online submission

- Thorough peer review

- Inclusion in PubMed and all major indexing services

- Maximum visibility for your research

Submit your manuscript at www.biomedcentral.com/submit 